\title{
Comparative Analysis of Age Dynamics of Average Values of Body Dimensions in Children from Birth to 7 years
}

\author{
Vasily E. Deryabin ${ }^{1)}$, Valentina M. Krans ${ }^{2)}$ and Tatiana K. Fedotova ${ }^{2)}$ \\ 1) Department of Anthropology, Biological Faculty, Moscow State University, Moscow, Russia \\ 2) Institute and Museum of Anthropology, Moscow State University, Moscow, Russia
}

\begin{abstract}
Mean values of different body dimensions in different age cohorts of children make it possible to learn a lot about their dynamic changes. Their comparative analysis, as is usually practiced, in fact leads to a simple description of changes in measurement units $(\mathrm{mm}$ or $\mathrm{cm})$ at the average level of some body dimension during a shorter or longer period of time. To estimate comparative intensity of the growth process of different body dimensions, the authors use the analogue of Mahalanobis distance, the so-called Kullback divergence (1967), which does not demand stability of dispersion or correlation coefficients of dimensions in compared cohorts of children. Most of the dimensions, excluding skinfolds, demonstrate growth dynamics with gradually reducing increments from birth to 7 years. Body length has the highest integrative increment, leg length about $94 \%$ of body length, body mass $77 \%$, and trunk and extremities circumferences $56 \%$. Skinfolds have a non-monotonic pattern of accumulated standardized increments with some increase until 1-2 years of age. J Physiol Anthropol Appl Human Sci 24(4): 487-491, $2005 \mathrm{http} / /$ www.jstage.jst.go.jp/browse/jpa
\end{abstract}

[DOI: $10.2114 /$ jpa.24.487]

Keywords: Kullback divergence, accumulated standardized increments, monotonic and non-monotonic patterns of growth changes

\section{Introduction}

Mean values of different body dimensions in different age cohorts of children make it possible to learn a lot about their dynamic changes. Their comparative analysis, as is usually practiced, in fact leads to a simple description of changes in measurement units $(\mathrm{mm}$ or $\mathrm{cm})$ at the average level of some body dimension during a shorter or longer period of time. Such an approach does not allow us to estimate comparative intensity of growth of different body dimensions, as they may have different measurement units, absolute average levels and variation scale.

One of the methods of studying the comparative degree of growth changes for different body dimensions is to express the dynamic series of each dimension in $k$ moments of time $X_{1}, X_{2}$, $\ldots, X_{k}$ in any standardized form. Such standardization is realized very often in the form of age series of proportions $X_{1} / X_{0}, \quad X_{2} / X_{0}, \ldots, X_{k} / X_{0}$, where $X_{0}$ is the standardizing dimension, usually the meaning of dimension from the beginning of the series $X_{1}$, or the final meaning of the series $X_{k}$. In the first case $\left(X_{0}=X_{1}\right)$ dimensions of a standardized row allow us to estimate the average increase of the dimension during the analyzed time interval. In the second case $\left(X_{0}=X_{k}\right)$ standardized file allows us to estimate the part of final dimension $X_{k}$ reached by the measurement at some period of time. The second case of standardization served as the basis of the well known Scammon (1930) diagram, describing basic growth types of different body systems. Such an approach allows us to estimate only comparative levels from initial or definite values, reached by different dimensions at some period of time, but does not characterize the comparative growth dynamics of different body dimensions.

Another biometrical method, based on more precise estimation of growth increments during some time interval, seems to be more valid. Let mean values of $m$ number of dimensions in the 1 st moment of time form the vector $\boldsymbol{X}_{1}=\left[X_{11}, X_{12}, \ldots, X_{1 m}\right]$, in the 2nd moment $\boldsymbol{X}_{2}=\left[X_{21}, X_{22}, \ldots\right.$, $\left.X_{2 m}\right]$. Then according to standard procedures of multivariate statistics a combined scale of dimensions divergence in two moments of time may be estimated by means of specially used in such cases Mahalanobis distance $D_{m}{ }^{2}=\left(\boldsymbol{X}_{1}-\boldsymbol{X}_{2}\right)^{\prime} \boldsymbol{S}^{-1}$ $\left(\boldsymbol{X}_{1}-\boldsymbol{X}_{2}\right)$, where $\boldsymbol{S}^{-1}$ is a matrix , inverse to a covariation matrix, including all dispersions and covariations of all paired combinations of dimensions. In case of a single dimension $X$, vectors of means $\boldsymbol{X}_{1}$ and $\boldsymbol{X}_{2}$ will be just mean values $\boldsymbol{X}_{1}$ and $\boldsymbol{X}_{2}$, a covariation matrix will be its intragroup dispersion $S^{2}$, while the Mahalanobis distance will have its most simple special case $D_{m}{ }^{2}=\left(X_{1}-X_{2}\right)^{2} / S^{2}$. Thus, a biometrically valid measure of the average level of any dimension during some period of time 
is a well known standardized difference of average values, expressed in parts of standard deviation. Due to this fact it will be completely comparable for all possible body dimensions.

At the same time, the empirically fixed fact of significant growth changes of the intragroup variation and correlation values of anthropometric dimensions in children of different ages (Borovka, 1928; Berkson, 1929; Brodovskaya, 1934; Aron, 1940; Solovjeva, 1964, 1965; Uryson, 1973; Vlastovsky, 1976; Deryabin et al., 2004) imposes substantial difficulties on the application of this method. The essence of these difficulties lies in the impossibility of operating with some unified level of standard deviation of dimension for a more or less prolonged time interval.

One of the possible solutions to the problem is based on using for the measuring of accumulated value of average differences of dimensions in two moments of time the analogue of the Mahalanobis distance, which does not require stability of dispersions and correlation coefficients of dimensions in compared groups of children. One such analogue is the so-called Kullback divergence (1967). The simple form of this interval measure is $D_{k}^{2}=\left[\left(D_{m 1}{ }^{2}+D_{m 2}{ }^{2}\right)+\right.$ $\left.V\left(\boldsymbol{S}_{1}, \boldsymbol{S}_{2}\right)\right] / 2$, where $D_{m 1}^{2}$ и $D_{m 2}{ }^{2}$ is the Mahalanobis distance, found with account of covariation matrix in the first $D_{m 1}{ }^{2}=\left(\boldsymbol{X}_{1}-\boldsymbol{X}_{2}\right)^{\prime} \boldsymbol{S}_{1}^{-1}\left(\boldsymbol{X}_{1}-\boldsymbol{X}_{2}\right)$ and the second $D_{m 2}{ }^{2}=\left(\boldsymbol{X}_{1}-\right.$ $\left.\boldsymbol{X}_{2}\right)^{\prime} \boldsymbol{S}_{2}^{-1}\left(\boldsymbol{X}_{1}-\boldsymbol{X}_{2}\right)$ moments of time. $\boldsymbol{X}_{1}$ and $\boldsymbol{X}_{2}$ are sets of average values in the first and second moments of time, $\boldsymbol{S}_{1}$ and $\boldsymbol{S}_{2}$ are intragroup covariation matrixes for these moments of time, and $V\left(\boldsymbol{S}_{1}, \boldsymbol{S}_{2}\right)$ is a term depending only on differences of covariation matrixes determined by correlation coefficients and dispersions of dimensions at two age points. The value $V\left(\boldsymbol{S}_{1}\right.$, $\boldsymbol{S}_{2}$ ) does not depend on average levels of dimensions and is not of any interest to the problem of morphological differences determined by them. Then the most interesting part of the Kullback interval, depending on differences of average values sets, may be put as $\left(D_{m 1}{ }^{2}+D_{m 2}{ }^{2}\right) / 2$.

In case of only one dimension, $X$ vectors of means $\boldsymbol{X}_{1}$ and $\boldsymbol{X}_{2}$ will be mean values $\boldsymbol{X}_{1}$ and $\boldsymbol{X}_{2}$, while covariation matrixes will be intragroup dispersions $\boldsymbol{S}_{1}{ }^{2}$ and $\boldsymbol{S}_{2}{ }^{2}$. Then the term of the Kullback divergence, depending on differences of average levels, will have a simple form of its specific case

$$
D= \pm\left[\frac{\left(X_{1}-X_{2}\right)^{2}}{2 S_{1}^{2}}+\frac{\left(X_{1}-X_{2}\right)^{2}}{2 S_{2}^{2}}\right]^{1 / 2}
$$

where the symbol \pm becomes $(+)$ when $\boldsymbol{X}_{1}>\boldsymbol{X}_{2}$ and $(-)$ when $\boldsymbol{X}_{1}<\boldsymbol{X}_{2}$. $D$ values found for different dimensions will be abstract values, expressed in parts of their standard deviations. Thus, they will be completely comparable for all analyzed body dimensions irrespective of their measurement units, absolute values or degree of intragroup variation.

It follows, that for $k$ time moments $t_{1}, t_{2}, \ldots, t_{k-1}, t_{k}$ for any dimensions we may get a series of values of standardized increments of dimensions $D_{\left(t_{2}-t_{1}\right)}, D_{\left(t_{3}-t_{2}\right)}, \ldots, D_{\left(t_{k}-t_{(k-1)}\right)}$ reflecting standardized growth velocities of body dimension. To get an idea of the form of dynamic curve describing age changes of the dimension, but not of its velocity, we may get the series of accumulated values of these velocities $S D_{t_{1}}=0, S D_{t_{2}}=D_{\left(t_{2}-t_{1}\right)}$, $S D_{t_{3}}=D_{\left(t_{2}-t_{1}\right)}+D_{\left(t_{3}-t_{2}\right)}, \ldots, S D_{t k}=D_{\left(t_{2}-t_{1}\right)}+D_{\left(t_{3}-t_{2}\right)}+\ldots+D_{\left(t_{k}-t_{(k-1)}\right)}$. It will begin from a conventional zero level in the first moment of time $t_{1}$ and any of its further values will enable us to state the level of changes of the dimension, expressed in intragroup standard deviation, for the period of time passed. Final values $S D_{t k}$ found for different body dimensions will enable us to compare the levels of growth changes of these dimensions on a broad time interval from $t_{1}$ to $t_{k}$ points.

\section{Material and Methods}

The proposed method was applied for the sample of Moscow boys and girls measured in 1973-1974 by means of a cross-sectional study undertaken by V. M. Krans (1979). The time interval from the moment of birth through 2.5 years was divided into age and sex groups with one month intervals. The number of observations for each of these groups was 20-40 children. The comparatively small number of observations in these groups was compensated for by an analytic procedure of the smoothing of dynamic series of the total data set by the mean squares method. This was meant to improve the influence of random effect in different age-sex groups. Agesex groups had a 1-year interval through the period of 3 to 7 years, and the number of children for each age and sex group was 100 . Thus, we operated with 36 age groups for each sex. Calculation of the velocities of changes of values $D_{\left(t_{2}-t_{1}\right)}$, $D_{\left(t_{3}-t_{2}\right)} \ldots D_{\left(t_{k}-t_{(k-1)}\right)}$ and of the accumulated levels of these velocities $S D_{t_{2}}, S D_{t_{3}}, \ldots, S D_{t_{k}}$ was undertaken using special software by V. E. Deryabin (Deryabin et al., 2004) called CULBAC (for MS-DOS).

\section{Results}

The dynamic series of the accumulated standardized $S D_{t i}$ changes of different body dimensions for boys are presented in Figs. 1-4. They have the same patterns for girls.

For most of the analyzed body dimensions (Figs. 1-3) excluding skinfolds (Fig. 4) dynamic changes of accumulated levels of standardized velocities demonstrate growth with gradually reducing increments. According to Figs. 1 and 2, children from birth to 2 months demonstrate an extremely high monthly growth velocity of body length and body mass, exceeding one unit of the value of standard deviation of these dimensions through the given time interval. The extremely high velocity is registered until 2 months of age when its value is close to 1.5 . The analogue values for the leg length are just slightly less.

As mentioned above, the applied methods of standardized growth curves allows us to compare the velocity values and final levels of body increments measured in different units and having different absolute dimensions and variation levels. To get a more detailed picture, one may express values of accumulated standardized velocities found in the final age 


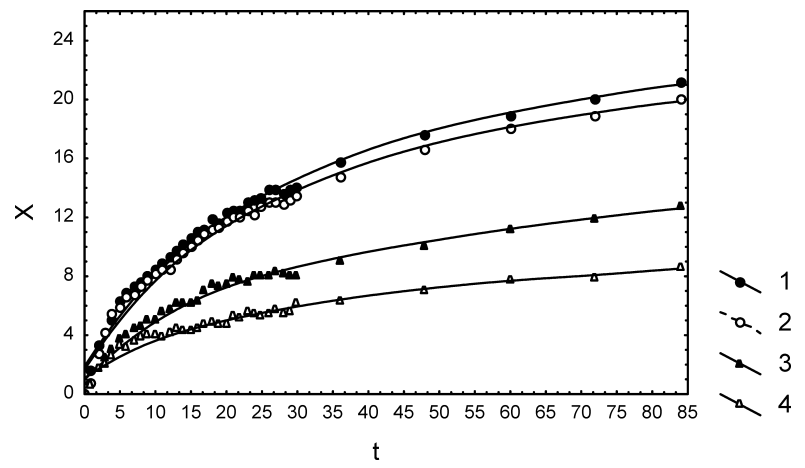

Fig. 1 Values of accumulated standardized velocities in Moscow boys: 1 -body length, 2-leg length, 3-biacromial diameter, 4-pelvic width, $t$-age in months.

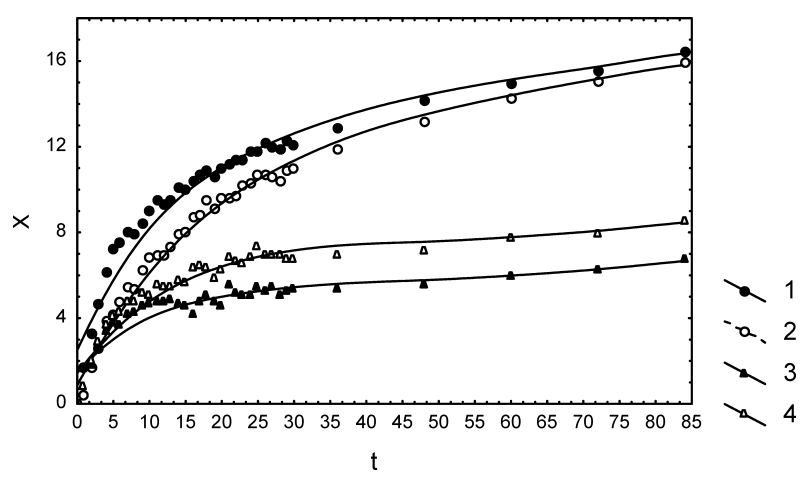

Fig. 2 Values of accumulated standardized velocities in Moscow boys: 1 -body mass, 2-foot length, 3-wrist circumference, 4-ankle circumference, $t$-age in months.

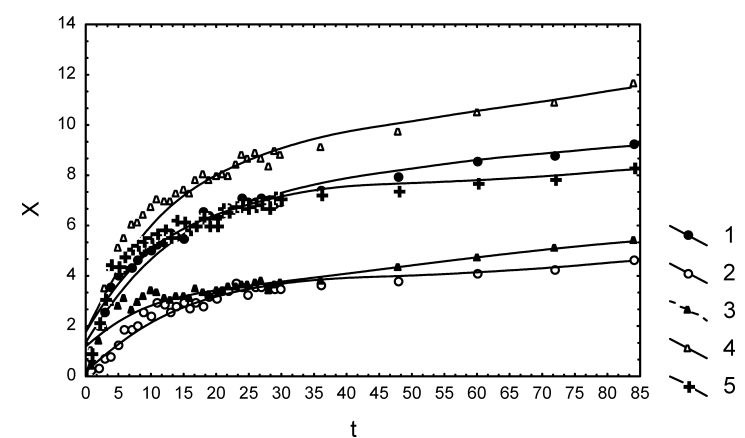

Fig. 3 Values of accumulated standardized velocities in Moscow boys: 1 -transversal chest diameter, 2-sagittal chest diameter, 3-neck circumference, 4-chest circumference, 5-waist circumference, $t$-age in months.

point ( 7 years) in the parts of the analogue levels of body length having maximum levels.

These parts of relative values of final changes for different body dimensions are represented in Table 1 . The comparative values of the total standardized increments of the dimensions reveal hardly any marked sex differences.

Leg length has the value of total standardized increment closest to body length, the level of its increment being 0.94 . It

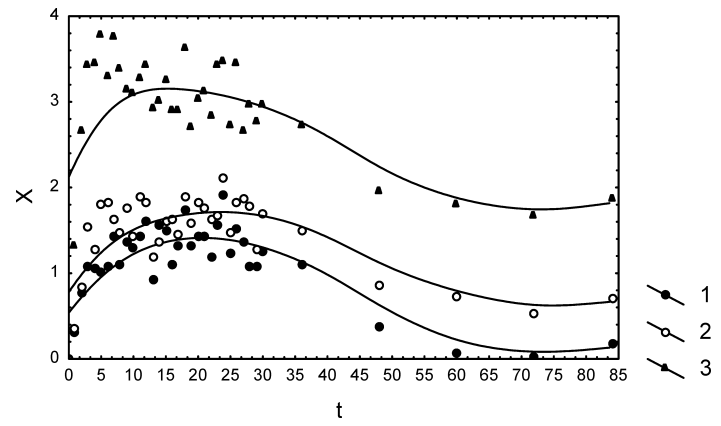

Fig. 4 Values of accumulated standardized velocities in Moscow boys: Skinfolds: 1 -subscapular, 2 -chest, 3 -bdomen, $t$-age in months.

Table 1 Proportion of accumulated standardized velocities of dimensions at 7 years of age, expressed in analogous levels of body length in boys (1) and girls (2)

\begin{tabular}{|c|c|c|c|}
\hline No. & Dimensions & 1 & 2 \\
\hline 1 & Body mass & 0.78 & 0.75 \\
\hline 2 & Body length & 1.00 & 1.00 \\
\hline 3 & Leg length & 0.95 & 0.93 \\
\hline 4 & Biacromial diameter & 0.60 & 0.63 \\
\hline 5 & Pelvic width & 0.41 & 0.45 \\
\hline 6 & Foot length & 0.75 & 0.78 \\
\hline 7 & Wrist circumference & 0.32 & 0.30 \\
\hline 8 & Ankle circumference & 0.40 & 0.43 \\
\hline 9 & Transversal chest diam. & 0.44 & 0.47 \\
\hline 10 & Sagittal chest diameter & 0.21 & 0.23 \\
\hline 11 & Neck circumference & 0.26 & 0.27 \\
\hline 12 & Chest circumference & 0.55 & 0.56 \\
\hline 13 & Waist circumference & 0.39 & 0.39 \\
\hline 14 & Arm circumference & 0.33 & 0.32 \\
\hline 15 & Forearm circumference & 0.40 & 0.40 \\
\hline 16 & Thigh circumference & 0.50 & 0.49 \\
\hline 17 & Shin circumference & 0.50 & 0.52 \\
\hline
\end{tabular}

means that the leg length value of total standardized increment from birth to 7 years of age equals $94 \%$ of the similar body length increment. These two dimensions have the highest growth velocities.

One more dimension having a rather high level of growth changes is foot length. Its total standardized changes equal about 0.77 of similar body length changes. Body mass shows 
the same value (0.77). Biacromial diameter, chest and shin circumferences have average levels of total growth changes through the $0-7$ interval, the values being $0.62,0.56$ and 0.51 , respectively.

Thigh circumference and transversal chest and pelvic diameters have even less levels of comparative total growth changes, their average levels being $0.50,0.46,0.43$. Forearm, waist, upper arm and wrist circumferences have very small levels of comparative increments, from 0.31 to 0.40 , while neck circumference and sagittal chest diameter are characterized by the smallest levels.

Skinfolds have principally different patterns of accumulated standardized increments dynamics through the 0-7 interval (Fig. 4). There is some increase of the level up to 1-2 years, followed by a consequent monotonous decrease up to 6 years of age and completion of the changes. The maximum level of accumulated standardized increments reached at 1-2 years of age equals 1.3-3.2 of standard deviation for different skinfolds, which is less than analogous values found for other dimensions.

\section{Discussion}

The dynamics of accumulated levels of standardized velocities for most of the body dimensions studied, excluding skinfolds, display a growth pattern with gradually decreasing increments. These results correspond with the growth patterns, revealed in the course of longitudinal studies of preschool children (reviews: Johnston, 1978; Tanner, 1994), though our study deals with cross-sectional data.

Skinfold patterns in our study correspond with the well known results of the Tanner and Whitehouse study (1975), demonstrating negative dynamics beginning from 1-2 years of age with minimum thickness at 5-7 years followed by a monotonous increase in their values till the end of the "second childhood". Further on, this process continues for girls, but settles down for boys at the beginning of the pubertal period. According to some longitudinal studies (review: Tanner, 1994) combined thickness of subscapular and triceps skinfolds increases from birth to 6 months and keeps almost stable till 9 months with a subsequent decrease of subcutaneous adipose tissue till 12 months. Figure 3 apparently represents the prolongation of this process.

Some other studies (Malina et al., 1974) of children with a definite nutrition deficiency from Guatemala register maximum values of subscapular and triceps skinfolds much earlier, at 3 months of age, with a subsequent decrease. Some groups of children (review: Johnston, 1978) do not show the distinct pattern of such dynamic changes (increase followed by decrease) in infancy.

\section{Conclusions}

Most of the body dimensions studied, excluding skinfolds, have a definite velocity pattern of changes of accumulated standardized values with monotonously decreasing increments.

Body length shows maximum total increments of growth changes, expressed in accumulated standardized values, among the whole sample of body dimensions through 0 to 7 years. Other skeletal dimensions have a wide range of total increments: from a maximal $94 \%$ for leg length to $31 \%$ for wrist circumference with an average level of $30-60 \%$. Body and extremities circumferences, dependent on muscle and fat development, have typical total increments about $30-40 \%$, body mass $77 \%$.

Skinfolds have a non-monotonic pattern of accumulated standardized increments through the 0-7 year interval, including some increase of dimension levels up to 1-2 years with a subsequent monotonous decrease up to 6 years with the later stabilization of these changes.

\section{References}

Aron DI (1940) Data for determination of proportions of children and adolescents aged 8-18 years. Ucheniye zapiski MGU 34: 103-125 [In Russian]

Berkson J (1929) Growth changes in physical correlationsheight, weight and chest circumference-males. Hum Biol1: 463-502

Borovka NV (1928) Correlations of basic anthropometric features depending on sex and age. Russkiy Antropologicheskiy zhurnal 17: 1-2, 66-77 [In Russian]

Brodovskaya VS (1934) Age dynamics of basic features of physical development. Medgiz, Moscow, 1-105 [In Russian]

Deryabin VE, Fedotova TK, Panasiuk TV (2004) Growth processes, stability and reconstructions of allocations of body dimensions of pre-school children. Manuscript deposited with VINITI No. 1610-B2004 dated 14.10.2004. Moscow, 1-229 [In Russian]

Johnston FE (1978) Somatic growth of the infant and preschool child. In Falkner F, Tanner JM eds. Human growth 2. Plenum Press, New York \& London, 91-116

Krans VM (1979) Principles of growth and development of children from birth to seven years of age, belonging to europeoid and mongoloid groups. Voprosy antropologii 61 : 70-82 [In Russian]

Kullback S (1967) Information theory and statistics. Nauka, Moscow, 1-408 [In Russian]

Malina RM, Habicht JP, Yarbrough C, Martorell R, Klein RE (1974) Skinfold thicknesses at seven sites in rural Guatemalan Ladino children birth through 7 years of age. Hum Biol 46: 453-462

Solovjeva VS (1964) Data on sexual maturation of Moscow schoolchildren and students. Voprosy antropologii 17: 35-61 [In Russian]

Solovjeva VS (1965) Comparative data on sexual maturation of Moscow and Tbilisi schoolgirls. Voprosy antropologii 19: 75-94 [In Russian]

Tanner JM (1994) Growth from birth to two: a critical review. Acta Med Auxol 26: 1-51 
Tanner JM, Whitehouse RH (1975) Revised standards for triceps and subscapular skinfolds in British children. Arch Dis Child 50: 142-145

Uryson AM (1973) Age dynamics of body dimensions of children and adolescents aged 4-18 years. In Miklashevskaya NN ed. Child growth and development. Moscow University publishing, Moscow, 4-53 [In Russian]

Scammon RE (1930) The measurement of body in childhood. In Harris JA, Jackson CM, Paterson DG, Scammon RE eds. Measurement of Man. University of Minnesota Press, Minneapolis, 173-215

Vlastovsky VG (1976) Acceleration of growth and development of children. Moscow University publishing, Moscow, 1-278 [In Russian]

Received: March 5, 2005

Accepted: March 26, 2005

Correspondence to: Dr. Tatiana Fedotova, Institute and $\mathrm{Mu}-$ seum of Anthropology, Moscow State University, Mokhovaja 11, Moscow 125009, Russia

Phone: +7-095-203-5067

Fax: +7-095-203-3554

e-mail: tatiana.fedotova@mail.ru 